\title{
ON NONNEGATIVE INTEGER MATRICES AND SHORT KILLING WORDS*
}

\author{
STEFAN KIEFER ${ }^{\dagger}$ AND CORTO N. MASCLE M $^{\ddagger}$
}

\begin{abstract}
Let $n$ be a natural number, and let $\mathcal{M}$ be a set of $n \times n$-matrices over the nonnegative integers such that the joint spectral radius of $\mathcal{M}$ is at most one. We show that if the zero matrix 0 is a product of matrices in $\mathcal{M}$, then there are $M_{1}, \ldots, M_{n^{5}} \in \mathcal{M}$ with $M_{1} \cdots M_{n^{5}}=0$. This result has applications in automata theory and the theory of codes. Specifically, if $X \subset \Sigma^{*}$ is a finite incomplete code, then there exists a word $w \in \Sigma^{*}$ of length polynomial in $\sum_{x \in X}|x|$ such that $w$ is not a factor of any word in $X^{*}$. This proves a weak version of Restivo's conjecture.
\end{abstract}

Key words. matrix semigroups, unambiguous automata, codes, Restivo's conjecture

AMS subject classifications. 20M35, 68Q45, 68R05

DOI. $10.1137 / 19 \mathrm{M} 1250893$

1. Introduction. Let $n \in \mathbb{N}$ and $\mathcal{M} \subseteq \mathbb{R}^{n \times n}$ be a finite set of matrices. The joint spectral radius of $\mathcal{M}$, denoted by $\rho(\mathcal{M})$, is defined by the following limit:

$$
\rho(\mathcal{M}):=\lim _{k \rightarrow \infty} \max \left\{\left\|M_{1} \cdots M_{k}\right\|^{1 / k}: M_{i} \in \mathcal{M}\right\} .
$$

This limit exists and does not depend on the chosen norm [7]. In this article we focus on nonnegative integer matrices: We assume $\mathcal{M} \subseteq \mathbb{N}^{n \times n}$, where $\mathbb{N}=\{0,1,2, \ldots\}$. Denote by $\overline{\mathcal{M}}$ the monoid (semigroup) generated by $\mathcal{M}$ under matrix multiplication, i.e., the set of products of matrices from $\mathcal{M}$. If $\overline{\mathcal{M}}$ is finite, then $\rho(\mathcal{M}) \leq 1$, but the converse does not hold [13].

In this article we show the following theorem.

TheOREM 1. Let $n \in \mathbb{N}$ and $\mathcal{M} \subseteq \mathbb{N}^{n \times n}$ be a finite set of nonnegative integer matrices with $\rho(\mathcal{M}) \leq 1$. Then there are $M_{1}, \ldots, M_{\ell} \in \mathcal{M}$ with $\ell \leq \frac{1}{16} n^{5}+\frac{15}{16} n^{4}$ such that the matrix product $M_{1} \cdots M_{\ell}$ has minimum rank among the matrices in $\overline{\mathcal{M}}$. Further, $M_{1}, \ldots, M_{\ell}$ can be computed in time polynomial in the description size of $\mathcal{M}$.

EXAMPLE 2. Let $n=3$ and $\mathcal{M}=\{A, B\}$, where

$$
A=\left(\begin{array}{lll}
0 & 0 & 1 \\
1 & 0 & 1 \\
0 & 0 & 0
\end{array}\right) \quad \text { and } B=\left(\begin{array}{lll}
0 & 0 & 0 \\
0 & 1 & 0 \\
0 & 1 & 0
\end{array}\right)
$$

Then $\overline{\mathcal{M}}$ is finite and $\rho(\mathcal{M})=1$. Further, the matrix product $A A A$ is the zero matrix and, hence, has rank 0 . No other product of length 3 yields the zero matrix.

* Received by the editors March 18, 2019; accepted for publication (in revised form) February 24, 2021; published electronically June 9, 2021. A preliminary version of this article appeared in STACS'19 under the title, On Finite Monoids over Nonnegative Integer Matrices and Short Killing Words. The current article is more self-contained and slightly generalizes the results by relaxing the finiteness condition to a condition on the joint spectral radius. In addition, we prove a more precise result related to Restivo's conjecture for finite codes.

https://doi.org/10.1137/19M1250893

Funding: The first author was supported by a Royal Society University Research Fellowship.

$\dagger$ University of Oxford, Oxford, OX1 2JD, UK (stekie@cs.ox.ac.uk, https://www.cs.ox.ac.uk/ people/stefan.kiefer/).

${ }^{\ddagger}$ Computer Science, ENS Paris-Saclay, 94235, Cachan CEDEX, France (corto.mascle@ens-parissaclay.fr). 
Let $\mathcal{M} \subseteq \mathbb{N}^{n \times n}$ be a finite set of nonnegative integer matrices. For notational convenience, throughout this article we associate to $\mathcal{M}$ a bijection $M: \Sigma \rightarrow \mathcal{M}$ and extend it to the monoid morphism $M: \Sigma^{*} \rightarrow \overline{\mathcal{M}}$, where $\Sigma^{*}=\bigcup_{i=0}^{\infty} \Sigma^{i}$ denotes the set of words over $\Sigma$. For a word $w \in \Sigma^{i}$, its length $|w|$ is $i$. We write $\varepsilon$ for the word of length 0 . We may write $M(\Sigma)$ for $\mathcal{M}$ and $M\left(\Sigma^{*}\right)$ for $\overline{\mathcal{M}}$ and $\rho(M)$ for $\rho(M(\Sigma)$ ). Then one may rephrase the main theorem as follows.

Theorem 1 (rephrased). Given $M: \Sigma \rightarrow \mathbb{N}^{n \times n}$ with $\rho(M) \leq 1$, one can compute in polynomial time a word $w \in \Sigma^{*}$ with $|w| \leq \frac{1}{16} n^{5}+\frac{15}{16} n^{4}$ such that $M(w)$ has minimum rank in $M\left(\Sigma^{*}\right)$.

The condition $\rho(M) \leq 1$ should be viewed in light of the following dichotomy [13]: if $\rho(M) \leq 1$, then $B(k):=\max \left\{\|M(w)\|: w \in \Sigma^{k}\right\}$ is in $O\left(k^{n}\right)$, i.e., $B(k)$ grows polynomially in $k$; if $\rho(M)>1$, then (by definition) $B(k)$ grows exponentially in $k$.

Automata definitions. A function $M: \Sigma \rightarrow\{0,1\}^{n \times n}$ is naturally associated with an automaton. A nondeterministic finite automaton (NFA) is a triple $\mathcal{A}=$ $(\Sigma, Q, \delta)$, where $\Sigma$ is a finite alphabet, $Q$ is a finite set of states, and $\delta: Q \times \Sigma \rightarrow 2^{Q}$ is a transition function (initial and final states do not play a role here). We extend $\delta$ in the usual way to $\delta: 2^{Q} \times \Sigma^{*} \rightarrow 2^{Q}$ by setting $\delta(P, a):=\bigcup_{q \in P} \delta(q, a)$ and $\delta(P, \varepsilon):=P$ and $\delta(P, w a):=\delta(\delta(P, w), a)$, where $P \subseteq Q$ and $a \in \Sigma$ and $w \in \Sigma^{*}$. A sequence $\psi=q_{0} a_{1} q_{1} a_{2} \cdots q_{n-1} a_{n} q_{n}$ with $q_{i} \in Q$ and $a_{i} \in \Sigma$ is called a path from $q_{0}$ to $q_{n}$ if $\delta\left(q_{i-1}, a_{i}\right) \ni q_{i}$ holds for all $i \in\{1, \ldots, n\}$. The word $a_{1} \cdots a_{n}$ is said to label the path $\psi$. Note that a word $w \in \Sigma^{*}$ labels a path from $p$ to $q$ if and only if $\delta(\{p\}, w) \ni q$. A word $w$ is called killing word if it does not label any path. Associate to $\mathcal{A}$ the monoid morphism $M_{\mathcal{A}}: \Sigma^{*} \rightarrow \mathbb{N}^{Q \times Q}$, where for all $a \in \Sigma$ we define $M_{\mathcal{A}}(a)(p, q)=1$ if $\delta(p, a) \ni q$ and 0 otherwise. Then, for any word $w \in \Sigma^{*}$ we have that $M_{\mathcal{A}}(w)(p, q)$ is the number of $w$-labelled paths from $p$ to $q$. In particular, $M_{\mathcal{A}}(w)$ is the zero matrix 0 if and only if $w$ is a killing word.

An NFA $\mathcal{A}=(\Sigma, Q, \delta)$ is called an unambiguous finite automaton (UFA) if for all states $p, q$ all paths from $p$ to $q$ are labelled by different words, i.e., for each word $w \in \Sigma^{*}$ there is at most one $w$-labelled path from $p$ to $q$. Call a monoid $\overline{\mathcal{M}} \subseteq \mathbb{N}^{n \times n}$ an unambiguous monoid of relations if $\overline{\mathcal{M}} \subseteq\{0,1\}^{n \times n}$. For every UFA $\mathcal{A}$ the monoid $M_{\mathcal{A}}\left(\Sigma^{*}\right)$ is an unambiguous monoid of relations, and every unambiguous monoid of relations can be viewed as generated by a UFA. UFAs play a central role in our proofs.

The mortality problem. Theorem 1 is related to the mortality problem for integer matrices: given $M: \Sigma \rightarrow \mathbb{Z}^{n \times n}$, is $0 \in M\left(\Sigma^{*}\right)$, i.e., can the zero matrix (which is defined to have rank 0) be expressed as a finite product of matrices in $M(\Sigma)$ ? Paterson [17] showed that the mortality problem for integer matrices is undecidable for $n=3$. It remains undecidable for $n=3$ with $|\Sigma|=7$ and for $n=21$ with $|\Sigma|=2$; see [10]. Mortality for $n=2$ is NP-hard [2] and not known to be decidable; see [18] for recent work on $n=2$.

The mortality problem for nonnegative matrices (even for matrices over the nonnegative reals) is much easier, as for each matrix entry it only matters whether it is zero or nonzero, so one can assume $M: \Sigma \rightarrow\{0,1\}^{n \times n}$. It follows that the mortality problem for nonnegative matrices is equivalent to the problem of whether an NFA has a killing word. The problem is PSPACE-complete [14], and there are examples where the shortest killing word has exponential length in the number of states of the automaton $[8,14]$. This implies that the assumption in Theorem 1 about the joint spectral radius $\rho(M)$ cannot be dropped. Whether $\rho(M) \leq 1$ indeed holds can be checked in polynomial time [13]. The condition is satisfied whenever $M\left(\Sigma^{*}\right)$ is finite. 
Whether or not $M\left(\Sigma^{*}\right)$ is finite can also be checked in polynomial time; see, e.g., [24] and the references therein. The authors are not aware of an easier proof of Theorem 1 under the stronger assumption that $M\left(\Sigma^{*}\right)$ is finite. If $\rho(M) \leq 1$, then the mortality problem for nonnegative integer matrices is solvable in polynomial time.

Proposition 3. Given $M: \Sigma \rightarrow \mathbb{N}^{n \times n}$ with $\rho(M) \leq 1$, one can decide in polynomial time whether $0 \in M\left(\Sigma^{*}\right)$.

Proposition 3 is implied by Theorem 1, but has an easier proof.

Short killing words for unambiguous finite automata. Proposition 3 provides a polynomial-time procedure for checking whether a UFA has a killing word. Define $\rho$ as the spectral radius of the rational matrix $\frac{1}{|\Sigma|} \sum_{a \in \Sigma} M(a)$. One can show that $\rho<1$ if $\mathcal{A}$ has a killing word, and $\rho=1$ otherwise (Lemma 8). Proposition 3 then follows from the fact that one can compare $\rho$ with 1 in polynomial time. Thus the spectral radius tells whether there exists a killing word, but does not provide a killing word. Neither does this method imply a polynomial bound on the length of a minimal killing word, let alone a polynomial-time algorithm for computing a killing word. Theorem 1, which is proved purely combinatorially, fills this gap: if there is a killing word, then one can compute a killing word of length $O\left(|Q|^{5}\right)$ in polynomial time. NP-hardness results for approximating the length of a shortest killing word were proved in [20], even for the case $|\Sigma|=2$ and for partial DFAs, which are UFAs with $|\delta(p, a)| \leq 1$ for all $p \in Q$ and all $a \in \Sigma$. In fact, by combining our main result with [20, Theorem 17] the following problem is NP-complete: given an unambiguous automaton and a number $\ell \in \mathbb{N}$ in binary, does there exist a killing word of length at most $\ell$ ?

Short minimum-rank words. Define the rank of a UFA $\mathcal{A}=(\Sigma, Q, \delta)$ as the minimum rank of the matrices $M_{\mathcal{A}}(w)$ for $w \in \Sigma^{*}$. A word $w$ such that the rank of $M_{\mathcal{A}}(w)$ attains that minimum is called a minimum-rank word. Minimum-rank words have been very well studied for deterministic finite automata (DFAs). DFAs are UFAs with $|\delta(p, a)|=1$ for all $p \in Q$ and all $a \in \Sigma$. In DFAs of rank 1 , minimum-rank words are called synchronizing because $\delta(Q, w)$ is a singleton when $w$ is a minimum-rank word. It is the famous Cerný conjecture that whenever a DFA has a synchronizing word, then it has a synchronizing word of length at most $(n-1)^{2}$ where $n:=|Q|$. There are DFAs whose shortest synchronizing words have that length, but the best known upper bound is cubic in $n$; see [23] for a survey on the Černý conjecture.

In 1986 Berstel and Perrin generalized the Černý conjecture from DFAs to UFAs by conjecturing [3] that in any UFA a shortest minimum-rank word has length $O\left(n^{2}\right)$. They remarked that no polynomial upper bound was known. Then Carpi [5] showed the following.

TheOREM 4 (Carpi [5]). Let $\mathcal{A}=(\Sigma, Q, \delta)$ be a UFA of rank $r \geq 1$ such that the state transition graph of $\mathcal{A}$ is strongly connected. Let $n:=|Q| \geq 1$. Then $\mathcal{A}$ has a minimum-rank word of length at most $\frac{1}{2} r n(n-1)^{2}+(2 r-1)(n-1)$.

This implies an $O\left(n^{4}\right)$ bound for the case where $r \geq 1$. Carpi left open the case $r=0$, i.e., when a killing word exists. The main technical contribution of our paper concerns the case $r=0$. Combined with Carpi's theorem, Theorem 4, we then obtain Theorem 1. Based on our technical development, we also provide a short proof of a variant of Carpi's theorem, which suffices for our purposes and makes this article self-contained. Theorem 1 provides, to the best of the authors' knowledge, the first polynomial bound, $O\left(n^{5}\right)$, on the length of shortest minimum-rank words for UFAs. 


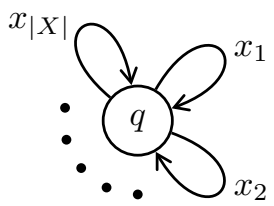

FIG. 1. Given a finite language $X \subseteq \Sigma^{*}$, the flower automaton $\mathcal{A}_{X}$ has one "petal" for each word $x \in X$. Thus $\delta(q, w) \ni q$ holds if and only if $w \in X^{*}$. If $X$ is a code, then $\mathcal{A}_{X}$ is unambiguous.

Restivo's conjecture. Let $X \subseteq \Sigma^{*}$ be a finite set of words over a finite alphabet $\Sigma$, and define $k:=\max _{x \in X}|x|$. A word $v \in \Sigma^{*}$ is called uncompletable in $X$ if there are no words $u, w \in \Sigma^{*}$ such that $u v w \in X^{*}$, i.e., $v$ is not a factor of any word in $X^{*}$. In 1981 Restivo [19] conjectured that if there exists an uncompletable word, then there is an uncompletable word of length at most $2 k^{2}$. This strong form of Restivo's conjecture was refuted in [9], with a lower bound of $5 k^{2}-O(k)$. See also [6] for more recent work and open problems related to Restivo's conjecture. The article [12] describes a sophisticated computer-assisted search for sets $X$ with long shortest uncompletable words. While these experiments did not formally disprove a quadratic upper bound in $k$, they seemed to hint at an exponential behavior in $k$. Indeed, in a recent preprint [16] a lower bound of $2^{k / 4} \cdot k / 4$ was given, refuting Restivo's conjecture fundamentally. The article [16] also provides a lower bound of $2^{\Omega\left(m^{1 / 5}\right)}$, where $m:=\sum_{x \in X}|x|$.

A set $X \subseteq \Sigma^{*}$ is called a code if every word $w \in X^{*}$ has at most one decomposition $w=x_{1} \cdots x_{\ell}$ with $x_{1}, \ldots, x_{\ell} \in X$. See [4] for a comprehensive reference on codes. For a finite code $X \subseteq \Sigma^{*}$ define $m:=\sum_{x \in X}|x|$. Given such $X$ one can construct a flower automaton [4, Chapter 4.2], which is a UFA $\mathcal{A}_{X}=(\Sigma, Q, \delta)$ with $m-|X|+1$ states; see Figure 1.

In this UFA any word is killing if and only if it is uncompletable in $X$. Hence Theorem 1 implies an $O\left(\mathrm{~m}^{5}\right)$ bound on the length of the shortest uncompletable word in a finite code. This proves a weak (note that $m^{5}$ may be much larger than $k^{2}$ ) version of Restivo's conjecture for finite codes. By adapting our main argument so that it exploits the special structure of flower automata, we get a better result.

Theorem 5. Let $X \subseteq \Sigma^{*}$ be a finite code that has an uncompletable word. Define $k:=\max _{x \in X}|x|$ and $m:=\sum_{x \in X}|x|$ and assume $k>0$. Then one can compute in polynomial time an uncompletable word of length at most $(k+1) k^{2}(m+2)(m+1)$.

This result does not contradict the work [16], as their sets $X$ are not codes. Contrasting the results of [16] with our Theorem 5, we highlight as an open problem the following version of Restivo's conjecture for finite codes: Does every finite code with an uncompletable word have an uncompletable word of length polynomial in $k$ ?

Is any product a short product? It was shown in [24] that if $M\left(\Sigma^{*}\right) \subseteq \mathbb{N}^{n \times n}$ is finite, then for every $w_{0} \in \Sigma^{*}$ there exists $w \in \Sigma^{*}$ with $|w| \leq\left[e^{2} n !\right]-2$ such that $M\left(w_{0}\right)=M(w)$. It was also shown in [24] that such a length bound cannot be smaller than $2^{n-2}$. In view of Theorem 1 one may ask if a polynomial length bound exists for low-rank matrices $M\left(w_{0}\right)$. The answer is no, even for unambiguous monoids of relations and even when $M\left(w_{0}\right)$ has rank 1 and 1 is the minimum rank in $M\left(\Sigma^{*}\right)$. 
THEOREM 6. There is no polynomial p such that the following holds:

Let $M: \Sigma^{*} \rightarrow\{0,1\}^{n \times n}$ be a monoid morphism. Let $w_{0} \in \Sigma^{*}$ be such that $M\left(w_{0}\right)$ has rank 1 , and let 1 be the minimum rank in $M\left(\Sigma^{*}\right)$. Then there is $w \in \Sigma^{*}$ with $|w| \leq p(n)$ such that $M\left(w_{0}\right)=M(w)$.

Thus, while Theorem 1 guarantees that some minimum-rank matrix in the monoid is a short product, this is not the case for every minimum-rank matrix in the monoid.

By how much could the $O\left(n^{5}\right)$ upper bound be improved? A synchronizing 0 -automaton is a DFA $\mathcal{A}=(\Sigma, Q, \delta)$ that has a state $0 \in Q$ and a word $w \in \Sigma^{*}$ such that $\delta(Q, w x)=\{0\}$ holds for all $x \in \Sigma^{*}$. The shortest such synchronizing words $w$ are exactly the shortest killing words in the partial DFA obtained from $\mathcal{A}$ by omitting all transitions into the state 0 . There exist synchronizing 0 -automata with $n$ states where the shortest synchronizing word has length $n(n-1) / 2$, and $\frac{n^{2}}{4}+\Omega(n)$ lower bounds exist even for synchronizing 0 -automata with $|\Sigma|=2[15,1]$. This implies that the $O\left(n^{5}\right)$ upper bound from Theorem 1 cannot be improved to $o\left(n^{2}\right)$, not even when a killing word exists. One might generalize the Černý conjecture by claiming Theorem 1 with an upper bound of $(n-1)^{2}$ (note that such a conjecture would concern minimum-rank words, not minimum nonzero-rank words). To the best of the authors' knowledge, this vast generalization of the Černý conjecture has not yet been refuted.

Organization of the article. In the remaining four sections we prove Proposition 3 and Theorems 1, 5, and 6 , respectively.

2. Proof of Proposition 3. Let $M: \Sigma \rightarrow \mathbb{N}^{n \times n}$ be such that $\rho(M) \leq 1$.

Towards a proof of Proposition 3, define the rational nonnegative matrix $A \in$ $\mathbb{Q}^{n \times n}$ by $A:=\frac{1}{|\Sigma|} \sum_{a \in \Sigma} M(a)$. Observe that for $k \in \mathbb{N}$ we have $A^{k}=\frac{1}{\left|\Sigma^{k}\right|} \sum_{w \in \Sigma^{k}}$ $M(w)$, i.e., $A^{k}$ is the average of the $M(w)$, where $w$ ranges over all words of length $k$. Define $\rho \geq 0$ as the spectral radius of $A$.

Lemma 7. We have $\rho \leq 1$.

Proof. By the Perron-Frobenius theorem, $A$ has a nonnegative eigenvector $u \in \mathbb{R}^{n}$ with $A u=\rho u$. So $A^{k} u=\rho^{k} u$. Thus $\max \left\{\|M(w)\|: w \in \Sigma^{k}\right\} \in \Omega\left(\rho^{k}\right)$. Hence $\rho \leq \rho(M) \leq 1$.

Lemma 8. We have $\rho<1$ if and only if there is $w \in \Sigma^{*}$ with $M(w)=0$.

Proof. Suppose $\rho<1$. Then $\lim _{k \rightarrow \infty} A^{k}=0$, and so there is $k \in \mathbb{N}$ such that the sum of all entries of $A^{k}$ is less than 1. It follows that there is $w \in \Sigma^{k}$ such that the sum of all entries of $M(w)$ is less than 1 . Since $M(w) \in \mathbb{N}^{n \times n}$, it follows that $M(w)=0$.

Conversely, suppose there is $w_{0} \in \Sigma^{*}$ with $M\left(w_{0}\right)=0$. Since $\rho(M) \leq 1$, by [13, Theorem 3] there exists $c>0$ such that $B(k):=\max \left\{\|M(w)\|: w \in \Sigma^{k}\right\} \leq c k^{n}$ holds for all $k \in \mathbb{N} \backslash\{0\}$. For any $k \in \mathbb{N}$ define $W(k):=\Sigma^{k} \backslash\left(\Sigma^{*} w_{0} \Sigma^{*}\right)$, i.e., $W(k)$ is the set of length- $k$ words that do not contain $w_{0}$ as a factor. Note that $M(w)=0$ holds for all $w \in \Sigma^{k} \backslash W(k)$. Since matrix norms are subadditive, it follows that $\left\|A^{k}\right\|$ is at most $\frac{|W(k)|}{\left|\Sigma^{k}\right|} \cdot B(k)$. On the other hand, for any $m \in \mathbb{N}$, if a word of length $m\left|w_{0}\right|$ is picked uniformly at random, then the probability of picking a word in $W\left(m\left|w_{0}\right|\right)$ is at most

$$
\left(1-\frac{1}{\left|\Sigma^{\left|w_{0}\right|}\right|}\right)^{m}
$$


thus

$$
\left\|A^{m\left|w_{0}\right|}\right\| \leq\left(1-\frac{1}{\left|\sum^{\left|w_{0}\right|}\right|}\right)^{m} c\left(m\left|w_{0}\right|\right)^{n}
$$

Hence $\lim _{k \rightarrow \infty} A^{k}=0$ and so $\rho<1$.

With these lemmas at hand, we can prove Proposition 3.

Proposition 3. Given $M: \Sigma \rightarrow \mathbb{N}^{n \times n}$ with $\rho(M) \leq 1$, one can decide in polynomial time whether $0 \in M\left(\Sigma^{*}\right)$.

Proof. By Lemma 8, it suffices to check whether $\rho<1$.

If $\rho<1$, then the linear system $A x=x$ does not have a nonzero solution. Conversely, if $\rho \geq 1$, then by Lemma 7 we have $\rho=1$ and thus, by the PerronFrobenius theorem, the linear system $A x=x$ has a (real) nonzero solution.

Hence it suffices to check if $A x=x$ has a nonzero solution. This can be done in polynomial time.

As remarked in section 1, this algorithm does not exhibit a word $w$ with $M(w)=0$, even when it proves the existence of such $w$.

3. Proof of Theorem 1. As before, let $M: \Sigma \rightarrow \mathbb{N}^{n \times n}$ be such that $\rho(M) \leq 1$. Call $M$ strongly connected if for all $i, j \in\{1, \ldots, n\}$ there is $w \in \Sigma^{*}$ with $M(w)(i, j) \geq$ 1. In subsection 3.1 we consider the case that $M$ is strongly connected. In subsection 3.2 we consider the general case.

3.1. Strongly connected. In this section we consider the case that $M$ is strongly connected and prove the following proposition, which extends Carpi's theorem, Theorem 4.

Proposition 9. Given $M: \Sigma \rightarrow \mathbb{N}^{n \times n}$ such that $\rho(M) \leq 1$ and $M$ is strongly connected, one can compute in polynomial time a word $w \in \Sigma^{*}$ with $|w| \leq \frac{1}{16} n^{5}+\frac{15}{16} n^{4}$ such that $M(w)$ has minimum rank in $M\left(\Sigma^{*}\right)$.

In the strongly connected case, $M\left(\Sigma^{*}\right)$ does not have numbers larger than 1 .

Lemma 10. We have $M\left(\Sigma^{*}\right) \subseteq\{0,1\}^{n \times n}$.

Proof. Suppose $M(v)(i, j) \geq 2$ for some $v \in \Sigma^{*}$. Since $M$ is strongly connected, there is $w \in \Sigma^{*}$ with $M(w)(j, i) \geq 1$. Hence $M(v w)(i, i) \geq 2$. It follows that $M\left((v w)^{k}\right)(i, i) \geq 2^{k}$ for all $k \in \mathbb{N}$, contradicting the assumption $\rho(M) \leq 1$.

Lemma 10 allows us to view the strongly connected case in terms of UFAs. Define a UFA $\mathcal{A}=(\Sigma, Q, \delta)$ with $Q=\{1, \ldots, n\}$ and $\delta(p, a) \ni q$ if and only if $M(a)(p, q)=1$. For the rest of the subsection we will mostly consider $Q$ as an arbitrary finite set of $n$ states. When there is no confusion, we may write $p w$ for $\delta(p, w)$ and $w q$ for $\{p \in Q: p w \ni q\}$. We extend this to $P w:=\bigcup_{p \in P} p w$ and $w P:=\bigcup_{p \in P} w p$. We say a state $p$ is reached by a word $w$ when $w p \neq \emptyset$, and a state $p$ survives a word $w$ when $p w \neq \emptyset$. Note that $Q w$ is the set of states that are reached by $w$, and $w Q$ is the set of states that survive $w$. Let $q_{1} \neq q_{2}$ be two different states. Then $q_{1}, q_{2}$ are called coreachable when there is $w \in \Sigma^{*}$ with $w q_{1} \cap w q_{2} \neq \emptyset$ (i.e., there is $p \in Q$ with $\left.p w \supseteq\left\{q_{1}, q_{2}\right\}\right)$, and they are called mergeable when there is $w \in \Sigma^{*}$ with $q_{1} w \cap q_{2} w \neq \emptyset$. For any $q \in Q$ we define $C(q)$ as the set of states coreachable with $q$. Also, define $c:=\max \left\{|q w|: q \in Q, w \in \Sigma^{*}\right\}$ and $m:=\max \left\{|w q|: w \in \Sigma^{*}, q \in Q\right\}$. The following lemma says that one can compute short witnesses for coreachability.

LEMma 11. If states $q \neq q^{\prime}$ are coreachable, then one can compute in polynomial time $w_{q, q^{\prime}} \in \Sigma^{*}$ with $\left|w_{q, q^{\prime}}\right| \leq \frac{1}{2}(n+2)(n-1)$ such that $q w_{q, q^{\prime}} \supseteq\left\{q, q^{\prime}\right\}$. 
Proof. Let $q \neq q^{\prime}$ be coreachable states. Then there are $p \in Q$ and $v \in \Sigma^{*}$ with $p v \supseteq\left\{q, q^{\prime}\right\}$. Since $M$ is strongly connected, there is $u \in \Sigma^{*}$ with $q u \ni p$, hence $q u v \supseteq\left\{q, q^{\prime}\right\}$. Define an edge-labelled directed graph $G=(V, E)$ with vertex set $V=\{\{r, s\}: r, s \in Q\}$ and edge set $E=\{(R, a, S) \in V \times \Sigma \times V: R a \supseteq S\}$. Since $q u v \supseteq\left\{q, q^{\prime}\right\}$, the graph $G$ has a path, labelled by $u v$, from $\{q\}$ to $\left\{q, q^{\prime}\right\}$. The shortest path from $\{q\}$ to $\left\{q, q^{\prime}\right\}$ has at most $|V|-1$ edges and is thus labelled with a word $w \in \Sigma^{*}$ with $|w| \leq|V|-1=\frac{1}{2} n(n+1)-1=\frac{1}{2}(n+2)(n-1)$. For this $w$ we have $q w \supseteq\left\{q, q^{\prime}\right\}$.

LEMma 12. For each $q \in Q$ one can compute in polynomial time a word $w_{q} \in \Sigma^{*}$ with $\left|w_{q}\right| \leq \frac{1}{2}(c-1)(n+2)(n-1)$ such that no state $q^{\prime} \neq q$ survives $w_{q}$ and is coreachable with $q$.

Proof. Let $q \in Q$. Consider the following algorithm:

1: $w:=\varepsilon$

2: while there is $q^{\prime} \in C(q)$ such that $q^{\prime}$ survives $w$ do

3: $\quad w:=w_{q, q^{\prime}} w$ (with $w_{q, q^{\prime}}$ from Lemma 11$)$

4: return $w_{q}:=w$

The following picture visualizes aspects of this algorithm:

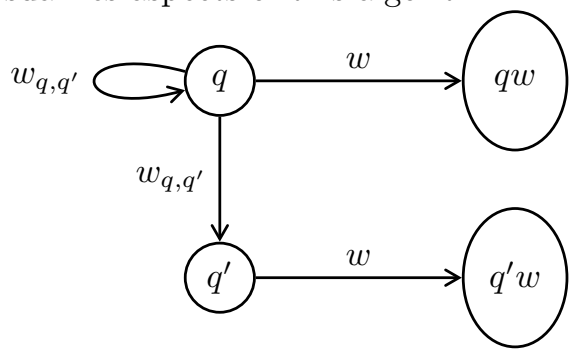

We argue that the computed word $w_{q}$ has the required properties. First, we show that the set $q w$ increases in each iteration of the algorithm. Indeed, let $w$ and $w_{q, q^{\prime}} w$ be the words computed by two subsequent iterations. Since $q w_{q, q^{\prime}} \supseteq\left\{q, q^{\prime}\right\}$, we have $q w_{q, q^{\prime}} w \supseteq q w \cup q^{\prime} w$. The set $q^{\prime} w$ is nonempty, as $q^{\prime}$ survives $w$. As can be read off from the picture above, the sets $q w$ and $q^{\prime} w$ are disjoint, as otherwise there would be two distinct paths from $q$ to a state in $q w \cap q^{\prime} w$, both labelled by $w_{q, q^{\prime}} w$, contradicting unambiguousness. It follows that $q w_{q, q^{\prime}} w \supsetneq q w$. Hence the algorithm must terminate.

Since in each iteration the set $q w$ increases by at least one element (starting from $\{q\})$, there are at most $c-1$ iterations. Hence $\left|w_{q}\right| \leq \frac{1}{2}(c-1)(n+2)(n-1)$. There is no state $q^{\prime} \neq q$ that survives $w_{q}$ and is coreachable with $q$, as otherwise the algorithm would not have terminated.

Lemma 13. One can compute in polynomial time words $z, y \in \Sigma^{*}$ such that

- $|z| \leq \frac{1}{4}(c-1)(n+2) n(n-1)$ and there are no two coreachable states that both survive $z$;

- $|y| \leq \frac{1}{4}(m-1)(n+2) n(n-1)$ and there are no two mergeable states that are both reached by $y$.

Proof. As the two statements are dual, we prove only the first one. Consider the following algorithm:

1: $w:=\varepsilon$

2: while there are coreachable $p, p^{\prime}$ that both survive $w$ do

3: $\quad q:=$ arbitrary state from $p w$

4: $\quad w:=w w_{q}\left(\right.$ with $w_{q}$ from Lemma 12)

Copyright $@$ ㅇ by SIAM. Unauthorized reproduction of this article is prohibited. 
5: $\operatorname{return} z:=w$.

We show that the set

$$
B:=\left\{p_{1} \in Q: \exists p_{2} \in C\left(p_{1}\right) \text { such that both } p_{1}, p_{2} \text { survive } w\right\}
$$

loses at least two states in each iteration. First, observe that

$$
B^{\prime}:=\left\{p_{1} \in Q: \exists p_{2} \in C\left(p_{1}\right) \text { such that both } p_{1}, p_{2} \text { survive } w w_{q}\right\}
$$

is clearly a subset of $B$.

Let $p \in B$ be the state from line 2 of the algorithm, and let $q \in p w$ be the state from the body of the loop. We claim that no $p^{\prime \prime} \in C(p)$ survives $w w_{q}$. Indeed, let $p^{\prime \prime} \in C(p)$. The following picture visualizes the situation:

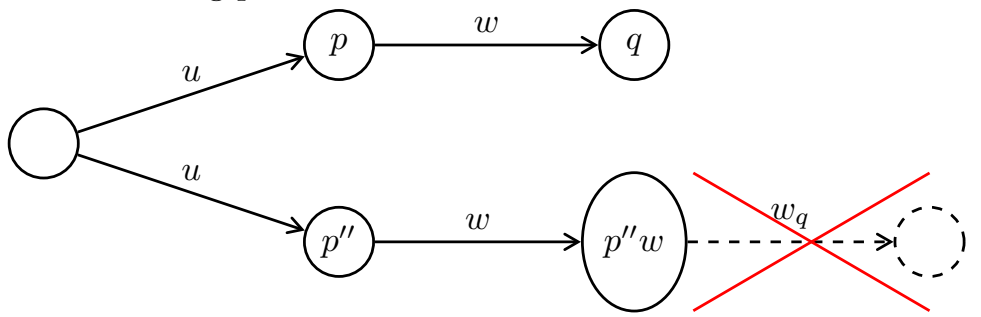

By unambiguousness and since $q \in p w$, we have $q \notin p^{\prime \prime} w$. By the definition of $w_{q}$ and since all states in $p^{\prime \prime} w$ are coreachable with $q$, we have $p^{\prime \prime} w w_{q}=\emptyset$, which proves the claim.

By the claim, we have $p \notin B^{\prime}$. Let $p^{\prime} \in B$ be the state $p^{\prime}$ from line 2 of the algorithm. We have $p^{\prime} \in C(p)$. By the claim, $p^{\prime}$ does not survive $w w_{q}$. Hence $p^{\prime} \notin B^{\prime}$.

So we have shown that the algorithm removes at least two states from $B$ in every iteration. Thus it terminates after at most $\frac{n}{2}$ iterations. Using the length bound from Lemma 12 we get $|z| \leq \frac{1}{4}(c-1)(n+2) n(n-1)$. There are no coreachable $q, q^{\prime}$ that both survive $z$, as otherwise the algorithm would not have terminated.

For the following development, let $q_{1}, \ldots, q_{k}$ be the states that are reached by $y$ and survive $z$ (with $y, z$ from Lemma 13); see Figure 2.

LEMmA 14. Let $1 \leq i<j \leq k$. Then $q_{i}, q_{j}$ are neither coreachable nor mergeable.

Proof. The proof is immediate from the properties of $y, z$ (Lemma 13).

The following lemma restricts sets of the form $q_{i} z x y z$ for $i \in\{1, \ldots, k\}$ and $x \in \Sigma^{*}$.

LEMmA 15. Let $i \in\{1, \ldots, k\}$ and $x \in \Sigma^{*}$. Then there is $j \in\{1, \ldots, k\}$ such that $q_{i} z x y z \subseteq q_{j} z$.

Proof. If $q_{i} z x y z=\emptyset$, then choose $j$ arbitrarily. Otherwise, let $q \in q_{i} z x y z$. Then $q$ is reached by $y z$, so there is $j$ with $q_{i} z x y \ni q_{j}$ and $q_{j} z \ni q$. We show that $q_{i} z x y z \subseteq q_{j} z$. To this end, let $q^{\prime} \in q_{i} z x y z$. Then $q^{\prime}$ is reached by $y z$, so there is $j^{\prime}$ with $q_{i} z x y \ni q_{j^{\prime}}$ and $q_{j^{\prime}} z \ni q^{\prime}$. Since $q_{i} z x y \supseteq\left\{q_{j}, q_{j^{\prime}}\right\}$ and $q_{j}, q_{j^{\prime}}$ are not coreachable (by Lemma 14), we have $j^{\prime}=j$. Hence $q_{j} z=q_{j^{\prime}} z \ni q^{\prime}$.

Provided that there is a killing word (which can be checked in polynomial time via Proposition 3), the following lemma asserts that for each $i \in\{1, \ldots, k\}$ one can efficiently compute a short word $x_{i}$ such that no state in $q_{i} z$ survives $x_{i} y z$. The proof hinges on a linear-algebra technique for checking equivalence of automata that are weighted over a field. The argument goes back to Schützenberger [21] and has often been rediscovered; see, e.g., [22]. 


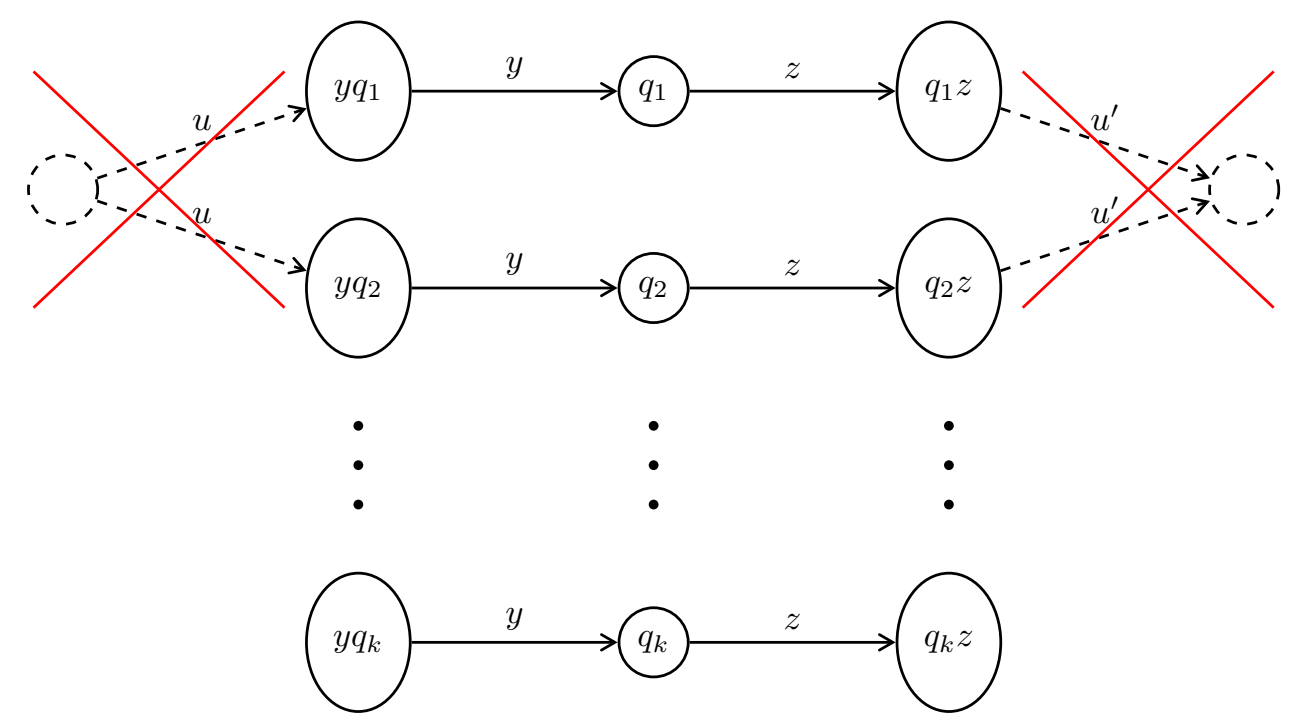

FIG. 2. The states $q_{1}, \ldots, q_{k}$ are neither coreachable nor mergeable.

Lemma 16. Suppose that $0 \in M\left(\Sigma^{*}\right)$. For each $i \in\{1, \ldots, k\}$ one can compute in polynomial time a word $x_{i} \in \Sigma^{*}$ with $\left|x_{i}\right| \leq n$ such that $q_{i} z x_{i} y z=\emptyset$.

Proof. Let $i \in\{1, \ldots, k\}$. Since $y\left\{q_{1}, \ldots, q_{k}\right\}$ are the only states to survive $y z$, it suffices to compute $x \in \Sigma^{*}$ with $|x| \leq n$ such that $q_{i} z x \cap y\left\{q_{1}, \ldots, q_{k}\right\}=\emptyset$.

Define $e \in\{0,1\}^{Q}$ as the characteristic row vector of $q_{i} z$, i.e., $e(q)=1$ if and only if $q \in q_{i} z$. Define $f \in\{0,1\}^{Q}$ as the characteristic column vector $y\left\{q_{1}, \ldots, q_{k}\right\}$. First, we show that for any $x \in \Sigma^{*}$ we have $e M(x) f \leq 1$. Towards a contradiction suppose $e M(x) f \geq 2$. Then there are two distinct $x$-labelled paths from $q_{i} z$ to $y\left\{q_{1}, \ldots, q_{k}\right\}$. It follows that there are two distinct $z x y$-labelled paths from $q_{i}$ to $\left\{q_{1}, \ldots, q_{k}\right\}$. By unambiguousness, these paths end in two distinct states $q_{j}, q_{j^{\prime}}$. But then $q_{j}, q_{j^{\prime}}$ are coreachable, contradicting Lemma 14. Hence we have shown that $e M(x) f \leq 1$ holds for all $x \in \Sigma^{*}$.

Define the (row) vector space

$$
V:=\left\langle(e M(x) \quad 1): x \in \Sigma^{*}\right\rangle \subseteq \mathbb{R}^{n+1},
$$

i.e., $V$ is spanned by the vectors $(e M(x) \quad 1)$ for $x \in \Sigma^{*}$. The vector space $V$ can be equivalently characterized as the smallest vector space that contains $\left(\begin{array}{ll}e & 1\end{array}\right)$ and is closed under multiplication with $\left(\begin{array}{rr}M(a) & 0 \\ 0 & 1\end{array}\right)$ for all $a \in \Sigma$. Hence the following algorithm computes a set $B \subseteq \Sigma^{*}$ such that $\{(e M(x) \quad 1): x \in B\}$ is a basis of $V$ :

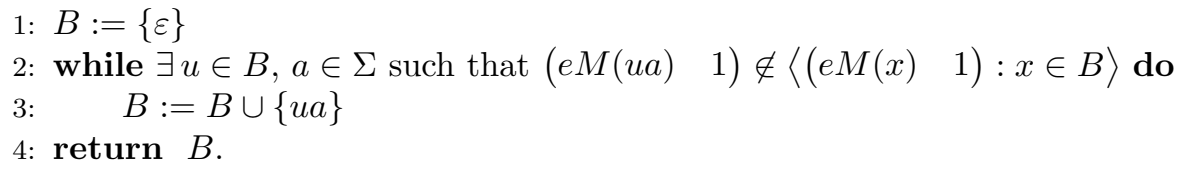

Observe that the algorithm performs at most $n$ iterations of the loop body, as every iteration increases the dimension of the space $\langle(e M(x) \quad 1): x \in B\rangle$ by 1 , but the dimension cannot grow larger than $n+1$. Hence $|x| \leq n$ holds for all $x \in B$. Since $M\left(w_{0}\right)=0$ holds for some $w_{0} \in \Sigma^{*}$ and hence $e M\left(w_{0}\right) f=0 \neq 1$, the space $V$ is not orthogonal to $\left(\begin{array}{c}f \\ -1\end{array}\right)$. So there exists $x \in B$ such that $e M(x) f \neq 1$. Since $e M(x) f \leq 1$, 
we have $e M(x) f=0$. Hence $q_{i} z x \cap y\left\{q_{1}, \ldots, q_{k}\right\}=\emptyset$.

Now we can prove the following lemma, which is our main technical contribution.

Lemma 17. Suppose that $0 \in M\left(\Sigma^{*}\right)$. One can compute in polynomial time a word $w \in \Sigma^{*}$ with $M(w)=0$ and $|w| \leq \frac{1}{16} n^{5}+\frac{15}{16} n^{4}$.

Proof. For any $1 \leq j<j^{\prime} \leq k$ the sets $q_{j} z$ and $q_{j^{\prime}} z$ are disjoint by Lemma 14 and nonempty. Hence any $P^{\prime} \subseteq Q$ has at most one set $P \subseteq\left\{q_{1}, \ldots, q_{k}\right\}$ with $P z=P^{\prime}$, which we call the generator of $P^{\prime}$. Note that all sets of the form $Q^{\prime} y z$ where $Q^{\prime} \subseteq Q$ have a generator. For any $i \in\{1, \ldots, k\}$, let $x_{i}$ be the word from Lemma 16, i.e., $q_{i} z x_{i} y z=\emptyset$. By Lemma 15, for any $j \in\{1, \ldots, k\}$ the generator of $q_{j} z x_{i} y z$ has at most one element. Thus, if $q_{i} \in P \subseteq\left\{q_{1}, \ldots, q_{k}\right\}$, then the generator, $P$, of $P z$ has strictly more elements than the generator of $P z x_{i} y z$.

Consider the following algorithm:

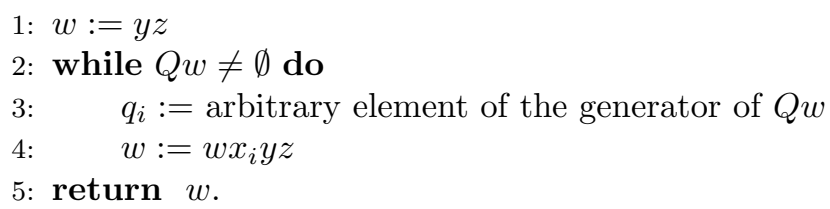

It follows from the argument above that the size of the generator of $Q w$ decreases in every iteration of the loop. Hence the algorithm terminates after at most $k$ iterations and computes a word $w$ such that $Q w=\emptyset$ and, using Lemmas 13 and 16,

$$
\begin{aligned}
|w| & \leq|y z|+k(n+|y z|) \leq n^{2}+(k+1)(|y|+|z|) \\
& \leq n^{2}+\frac{1}{4}(k+1)(c+m-2)(n+2) n(n-1) .
\end{aligned}
$$

Let $q, q^{\prime} \in Q$ and $u, u^{\prime} \in \Sigma^{*}$ such that $c=|q u|$ and $m=\left|u^{\prime} q^{\prime}\right|$. Clearly, $q u \cup u^{\prime} q^{\prime} \cup$ $\left\{q_{1}, \ldots, q_{k}\right\} \subseteq Q$, and it follows from the inclusion-exclusion principle:

$$
c+m+k \leq n+\left|q u \cap u^{\prime} q^{\prime}\right|+\left|q u \cap\left\{q_{1}, \ldots, q_{k}\right\}\right|+\left|\left\{q_{1}, \ldots, q_{k}\right\} \cap u^{\prime} q^{\prime}\right| .
$$

The sets $q u$ and $u^{\prime} q^{\prime}$ overlap in at most one state by unambiguousness. The sets $q u$ and $\left\{q_{1}, \ldots, q_{k}\right\}$ overlap in at most one state by Lemma 14 , and similarly for $\left\{q_{1}, \ldots, q_{k}\right\}$ and $u^{\prime} q^{\prime}$. It follows that $c+m+k \leq n+3$; thus $(k+1)+(c+m-2) \leq n+2$, and hence $(k+1)(c+m-2) \leq \frac{1}{4}(n+2)^{2}$. With the bound on $|w|$ from above we conclude that $|w| \leq n^{2}+\frac{1}{16}(n+2)^{3} n(n-1)$, which is bounded by $\frac{1}{16} n^{5}+\frac{15}{16} n^{4}$ for $n \geq 1$.

The following lemma, which rests on the properties of $y$ and $z$, provides an alternative to the use of Carpi's theorem, Theorem 4, in the proof of Proposition 9.

Lemma 18. Suppose that $0 \notin M\left(\Sigma^{*}\right)$. Then $M(y z)$ has minimum rank in $M\left(\Sigma^{*}\right)$ and this rank is $k$.

Proof. It follows from Lemma 14 that each row of $M(y z)$ is either the zero vector or the characteristic vector of some $q_{i} z$. As the sets $q_{i} z$ for $i \in\{1, \ldots, k\}$ are nonempty and pairwise disjoint, it follows that $M(y z)$ has rank $k$.

Suppose $x \in \Sigma^{*}$ is such that $M(x)$ has rank less than $k$. Then $M(y z x)$ has rank less than $k$. Since the sets $q_{i} z x$ for $i \in\{1, \ldots, k\}$ are pairwise disjoint, there is $i \in\{1, \ldots, k\}$ such that $q_{i} z x=\emptyset$. In order to show that $0 \in M\left(\Sigma^{*}\right)$ it suffices to show that for all $p \in Q$ and all $u \in \Sigma^{*}$ there is $w \in \Sigma^{*}$ such that $p u w=\emptyset$. Let $p \in Q$ and $u \in \Sigma^{*}$. If $p u=\emptyset$, then choose $w=\varepsilon$. Otherwise, let $v \in \Sigma^{*}$ be such that puvy $\ni q_{i}$. By Lemma 14, we have puvy $\cap\left\{q_{1}, \ldots, q_{k}\right\}=\left\{q_{i}\right\}$. Thus puvyz $=q_{i} z$ and puvyzx $=q_{i} z x=\emptyset$. Hence choose $w=v y z x$. 
To prove Proposition 9 we combine Lemma 17 with either Carpi's theorem, Theorem 4, or Lemma 18.

Proposition 9. Given $M: \Sigma \rightarrow \mathbb{N}^{n \times n}$ such that $\rho(M) \leq 1$ and $M$ is strongly connected, one can compute in polynomial time a word $w \in \Sigma^{*}$ with $|w| \leq \frac{1}{16} n^{5}+\frac{15}{16} n^{4}$ such that $M(w)$ has minimum rank in $M\left(\Sigma^{*}\right)$.

Proof. One can check in polynomial time whether $0 \in M\left(\Sigma^{*}\right)$; see Proposition 3. If yes, then the minimum rank is 0, and Lemma 17 gives the result. Otherwise, $0 \notin M\left(\Sigma^{*}\right)$, and Lemmas 13 and 18 give the result.

In the case $0 \notin M\left(\Sigma^{*}\right)$ one may alternatively use Carpi's theorem, Theorem 4. Indeed, the minimum rank $r$ is between 1 and $n$, and hence $n \geq 1$. Theorem 4 asserts the existence of a word $w$ such that $M(w)$ has rank $r$ and $|w| \leq \frac{1}{2} n^{4}-n^{3}+\frac{5}{2} n^{2}-3 n+1$, which is bounded by $\frac{1}{16} n^{5}+\frac{15}{16} n^{4}$ for $n \geq 1$. An inspection of Carpi's proof [5] shows that his proof is constructive and can be transformed into an algorithm that computes $w$ in polynomial time.

3.2. Not necessarily strongly connected. We prove Theorem 1 .

TheOREM 1 (rephrased). Given $M: \Sigma \rightarrow \mathbb{N}^{n \times n}$ with $\rho(M) \leq 1$, one can compute in polynomial time a word $w \in \Sigma^{*}$ with $|w| \leq \frac{1}{16} n^{5}+\frac{15}{16} n^{4}$ such that $M(w)$ has minimum rank in $M\left(\Sigma^{*}\right)$.

Proof. For any matrix $A$ denote by $r k(A)$ its rank. For $i, j \in\{1, \ldots, n\}$ write $i \rightarrow j$ if there is $u \in \Sigma^{*}$ such that $M(u)(i, j)>0$, and write $i \leftrightarrow j$ if $i \rightarrow j$ and $j \rightarrow i$. The relation $\leftrightarrow$ is an equivalence relation. Denote by $C_{1}, \ldots, C_{h} \subseteq\{1, \ldots, n\}$ its equivalence classes $(h \leq n)$. We can assume that whenever $i \in C_{k}$ and $j \in C_{\ell}$ and $i \rightarrow j$, then $k \leq \ell$. Hence, without loss of generality, $M(u)$ for any $u \in \Sigma^{*}$ has the following block-upper triangular form:

$$
M(u)=\left(\begin{array}{cccc}
M_{11}(u) & M_{12}(u) & \cdots & M_{1 h}(u) \\
0 & M_{22}(u) & \cdots & M_{2 h}(u) \\
\vdots & \vdots & \ddots & \vdots \\
0 & 0 & \cdots & M_{h h}(u)
\end{array}\right),
$$

where $M_{i i}(u) \in \mathbb{N}^{\left|C_{i}\right| \times\left|C_{i}\right|}$ for all $i \in\{1, \ldots, h\}$. For $i \in\{1, \ldots, h\}$ define $r_{i}:=$ $\min _{u \in \Sigma^{*}} r k\left(M_{i i}(u)\right)$. For any $u \in \Sigma^{*}$ we have $r k(M(u)) \geq \sum_{i=1}^{h} r k\left(M_{i i}(u)\right)$ (see, e.g., [11, Chapter 0.9.4]). It follows that the minimum rank among the matrices in $M\left(\Sigma^{*}\right)$ is at least $\sum_{i=1}^{h} r_{i}$.

Let $w_{1}, \ldots, w_{h} \in \Sigma^{*}$ be the words from Proposition 9 for $M_{11}, \ldots, M_{h h}$, respectively, so that $r k\left(M_{i i}\left(w_{i}\right)\right)=r_{i}$ holds for all $i \in\{1, \ldots, h\}$. Define $w:=w_{1} \cdots w_{h}$. Then we have

$$
|w| \leq \sum_{i=1}^{h}\left|w_{i}\right| \leq \sum_{i=1}^{h} \frac{1}{16}\left|C_{i}\right|^{5}+\frac{15}{16}\left|C_{i}\right|^{4} \leq \frac{1}{16} n^{5}+\frac{15}{16} n^{4} .
$$

It remains to show that $r k(M(w)) \leq \sum_{i=1}^{h} r_{i}$. It suffices to prove that $r k\left(M_{k}\left(w_{1}\right.\right.$ $\left.\left.\cdots w_{k}\right)\right) \leq \sum_{i=1}^{k} r_{i}$ holds for all $k \in\{1, \ldots, h\}$, where $M_{k}(u)$ for any $u \in \Sigma^{*}$ is the principal submatrix obtained by restricting $M(u)$ to the rows and columns corresponding to $\bigcup_{i=1}^{k} C_{i}$. We proceed by induction on $k$. For the base case, $k=1$, we have $r k\left(M_{1}\left(w_{1}\right)\right)=r k\left(M_{11}\left(w_{1}\right)\right)=r_{1}$. For the induction step, let $1<k \leq h$. Then 
there are matrices $A_{1}, A_{2}, B_{1}, B_{2}$ such that

$$
\begin{aligned}
M_{k}\left(w_{1} \cdots w_{k}\right) & =M_{k}\left(w_{1} \cdots w_{k-1}\right) M_{k}\left(w_{k}\right) \\
& =\left(\begin{array}{cc}
M_{k-1}\left(w_{1} \cdots w_{k-1}\right) & A_{1} \\
0 & A_{2}
\end{array}\right)\left(\begin{array}{cc}
B_{1} & B_{2} \\
0 & M_{k k}\left(w_{k}\right)
\end{array}\right) \\
& =\left(\begin{array}{cc}
M_{k-1}\left(w_{1} \cdots w_{k-1}\right) \\
0
\end{array}\right)\left(\begin{array}{ll}
B_{1} & B_{2}
\end{array}\right)+\left(\begin{array}{l}
A_{1} \\
A_{2}
\end{array}\right)\left(\begin{array}{ll}
0 & \left.M_{k k}\left(w_{k}\right)\right) .
\end{array}\right.
\end{aligned}
$$

By the induction hypothesis, we have $r k\left(M_{k-1}\left(w_{1} \cdots w_{k-1}\right)\right) \leq \sum_{i=1}^{k-1} r_{i}$. Further, we have $r k\left(M_{k k}\left(w_{k}\right)\right)=r_{k}$. So the ranks of the two summands in (3.1) are at most $\sum_{i=1}^{k-1} r_{i}$ and $r_{k}$, respectively. Since for any matrices $A, B$ it holds that $r k(A+$ $B) \leq r k(A)+r k(B)$, we conclude that $r k\left(M_{k}\left(w_{1} \cdots w_{k}\right)\right) \leq \sum_{i=1}^{k} r_{i}$, completing the induction proof.

\section{Proof of Theorem 5 .}

Theorem 5. Let $X \subseteq \Sigma^{*}$ be a finite code that has an uncompletable word. Define $k:=\max _{x \in X}|x|$ and $m:=\sum_{x \in X}|x|$ and assume $k>0$. Then one can compute in polynomial time an uncompletable word of length at most $(k+1) k^{2}(m+2)(m+1)$.

Consider the flower automaton associated to $X$, which is a UFA $\mathcal{A}=(\Sigma, Q, \delta)$ with $n=|Q|=m-|X|+1$ states. The uncompletable words in $X$ are exactly the killing words in $\mathcal{A}$. Towards a proof of Theorem 5 we first focus on computing a short killing word in $\mathcal{A}$.

To this end we optimize the construction from subsection 3.1 for flower automata. Denote by $0 \in Q$ the "central" state of $\mathcal{A}$ around which the petals are built. For each $q \in Q$ fix a word $u_{q} \in \Sigma^{*}$ such that $q u_{q}=\{0\}$ and $\left|u_{q}\right| \leq k-1$. The following lemma bounds the size of certain sets of states that survive long words.

Lemma 19. Let $w \in \Sigma^{*}$ with $|w| \geq k-1$. Then for all $p \in Q$ and all $v \in \Sigma^{*}$ we have $|p v \cap w Q| \leq k$, i.e., at most $k$ states of pv survive $w$.

Proof. Towards a contradiction, suppose $|p v \cap w Q|>k$. By the pigeonhole principle, there are two different states $q_{1}, q_{2}$ with $q_{1}, q_{2} \in p v \cap w Q$ such that $\left|u_{q_{1}}\right|=\left|u_{q_{2}}\right|$. Since $q_{1}, q_{2}$ both survive $w$, where $|w| \geq k-1$, both $u_{q_{1}}$ and $u_{q_{2}}$ are prefixes of $w$. Hence $u_{q_{1}}=u_{q_{2}}$. It follows that $q_{1} u_{q_{1}}=\{0\}=q_{2} u_{q_{1}}$, i.e., $q_{1}, q_{2}$ are mergeable. But $q_{1}, q_{2} \in p v$ are also coreachable, contradicting unambiguousness.

The following lemma adapts Lemma 12.

LEMma 20. For each $q \in Q$ one can compute in polynomial time a word $w_{q} \in \Sigma^{*}$ with $\left|w_{q}\right| \leq \frac{1}{2}(k-1)(n+2) n$ such that no state $q^{\prime} \neq q$ survives $w_{q}$ and is coreachable with $q$.

Proof. We use the same algorithm as in the proof of Lemma 12, except that we initialize $w$ not to $\varepsilon$ but to some $w_{\text {init }} \in \Sigma^{k-1}$ with $q w_{\text {init }} \neq \emptyset$. Such $w_{\text {init }}$ exists, as $q$ is on some cycle. Let $\ell \in \mathbb{N}$ be the number of iterations of the loop in the algorithm. The computed word $w_{q}$ has the form $w_{q, q_{\ell}} \cdots w_{q, q_{1}} w_{\text {init }}$ for some states $q_{1}, \ldots, q_{\ell} \in Q$. It follows from the proof of Lemma 12 that for all $i \in\{1, \ldots, \ell\}$ we have $q w_{q, q_{i}} \cdots w_{q, q_{1}} w_{\text {init }} \supsetneq q w_{q, q_{i-1}} \cdots w_{q, q_{1}} w_{\text {init }}$. Hence also $q w_{q, q_{i}} \cdots w_{q, q_{1}} \cap$ $w_{\text {init }} Q \supsetneq q w_{q, q_{i-1}} \cdots w_{q, q_{1}} \cap w_{\text {init }} Q$. Since $q \in w_{\text {init }} Q$, it follows that $\mid q w_{q, q_{\ell}} \cdots w_{q, q_{1}}$ $\cap w_{\text {init }} Q \mid \geq \ell+1$. By Lemma 19 it follows that $\ell+1 \leq k$. Hence, using Lemma 11 we obtain $\left|w_{q}\right|=\left|w_{q, q_{\ell}} \cdots w_{q, q_{1}} w_{\text {init }}\right| \leq \frac{1}{2}(k-1)(n+2)(n-1)+(k-1) \leq \frac{1}{2}(k-1)(n+$ 2)n. 
The following lemma adapts Lemma 13.

LEMMA 21. One can compute in polynomial time words $z, y \in \Sigma^{*}$ such that

- $|z| \leq \frac{1}{2} k(k-1)(n+2)(n+1)$ and there are no two coreachable states that both survive $z$;

- $|y| \leq \frac{1}{2} k(k-1)(n+2)(n+1)$ and there are no two mergeable states that are both reached by $y$.

Proof. We use the same algorithm as in the proof of Lemma 13, except that we initialize $w$ not to $\varepsilon$ but to an arbitrary $w_{\text {init }} \in \Sigma^{k-1}$, and that for $w_{q}$ we use Lemma 20 instead of Lemma 12:

1: $w:=w_{\text {init }}$

2: while there are coreachable $p, p^{\prime}$ that both survive $w$ do

3: $\quad q:=$ arbitrary state from $p w$

4: $\quad w:=w w_{q}\left(\right.$ with $w_{q}$ from Lemma 20$)$

5: return $z:=w$.

Consider a state $p \in Q$ picked in some iteration of the loop, i.e., $p$ survives the (current) word $w$. We claim that no state $\bar{p}$ with $\left|u_{\bar{p}}\right|=\left|u_{p}\right|$ will be picked in any future iteration. Indeed, let $\bar{p} \in Q$ be with $\left|u_{\bar{p}}\right|=\left|u_{p}\right|$ such that $\bar{p}$ survives a future $w$. Then $\bar{p}$ survives the current $w$. Since $\left|u_{p}\right|=\left|u_{\bar{p}}\right|$ and $p, \bar{p}$ both survive $w$ with $|w| \geq k-1$, we have $u_{p}=u_{\bar{p}}$ and this word is a prefix of $w$. It follows that $p w=\bar{p} w$; thus $q \in \bar{p} w$, where $q$ is the state from line 3 . Suppose $\bar{p}^{\prime}$ is an arbitrary state that is coreachable with $\bar{p}$. Then the states in $\bar{p}^{\prime} w$ are coreachable with $q$. Thus, $\bar{p}^{\prime} w w_{q}=\emptyset$ and so $\bar{p}^{\prime}$ does not survive any future $w$. It follows that $\bar{p}$ will not be picked in any future iteration.

Since for all $p \in Q$ we have $\left|u_{p}\right| \in\{0, \ldots, k-1\}$, the algorithm performs at most $k$ loop iterations. Hence, using Lemma 20, the computed word $z$ has length at most $k \cdot \frac{1}{2}(k-1)(n+2) n+(k-1) \leq \frac{1}{2} k(k-1)(n+2)(n+1)$. The argument for $y$ is similar.

The following lemma adapts Lemma 17.

LEMma 22. One can compute in polynomial time a killing word of length at most $(k+1) k^{2}(n+2)(n+1)$.

Proof. Let $z, y$ be the words from Lemma 21. We can assume that $|z| \geq k-1$. First, we argue that there are at most $k$ states that are reached by $y$ and survive $z$. Towards a contradiction, suppose otherwise. By the pigeonhole principle, there are two distinct states $q, q^{\prime} \in Q$ that are reached by $y$ and survive $z$ and satisfy $\left|u_{q}\right|=\left|u_{q^{\prime}}\right|$. Since $|z| \geq k-1$, it follows that $u_{q}=u_{q^{\prime}}$ is a prefix of $z$; thus $q, q^{\prime}$ are mergeable. But $q, q^{\prime}$ are reached by $y$, contradicting the definition of $y$.

It follows that the $k$ from subsection 3.1 is at most the $k$ from this section. Mirroring exactly the proof of Lemma 17 and using Lemma 21, we obtain a killing word $w$ of length at most

$$
\begin{aligned}
|w| & \leq|y z|+k(n+|y z|) \leq n^{2}+(k+1)(|y|+|z|) \\
& \leq n^{2}+(k+1) k(k-1)(n+2)(n+1) \leq(k+1) k^{2}(n+2)(n+1) .
\end{aligned}
$$

Finally we prove Theorem 5 .

Proof of Theorem 5. Since $k>0$, it follows that $|X| \geq 1$ and thus $n=m-|X|+$ $1 \leq m$. The result follows from Lemma 22 .

\section{Proof of Theorem 6.}

Copyright $@$ by SIAM. Unauthorized reproduction of this article is prohibited. 


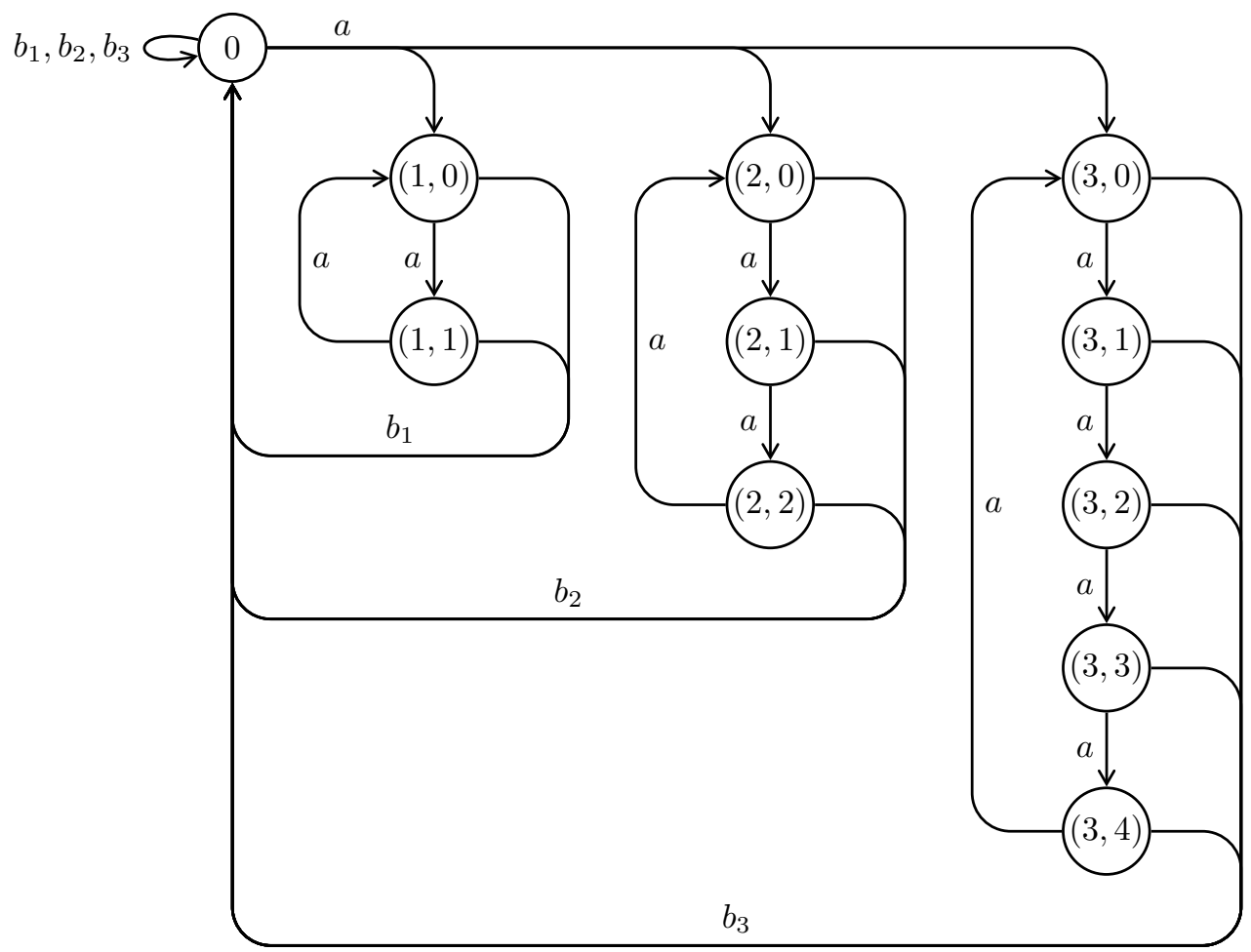

FIG. 3. Automaton representation of $M$ for $m=3$.

THEOREM 6. There is no polynomial $p$ such that the following holds:

Let $M: \Sigma^{*} \rightarrow\{0,1\}^{n \times n}$ be a monoid morphism. Let $w_{0} \in \Sigma^{*}$ be such that $M\left(w_{0}\right)$ has rank 1 , and let 1 be the minimum rank in $M\left(\Sigma^{*}\right)$. Then there is $w \in \Sigma^{*}$ with $|w| \leq p(n)$ such that $M\left(w_{0}\right)=M(w)$.

Proof. Denote by $p_{i}$ the $i$ th prime number (so $p_{1}=2$ ). Let $m \geq 1$. Define the following:

$$
\begin{aligned}
\Sigma & :=\left\{a, b_{1}, \ldots, b_{m}\right\}, \\
Q_{i} & :=\left\{(i, 0),(i, 1), \ldots,\left(i, p_{i}-1\right)\right\} \quad \text { for every } i \in\{1, \ldots, m\}, \\
Q & :=\{0\} \cup \bigcup_{i=1}^{m} Q_{i} .
\end{aligned}
$$

Further, define a monoid morphism $M: \Sigma^{*} \rightarrow \mathbb{N}^{Q \times Q}$ by setting for all $i \in\{1, \ldots, m\}$

$$
\begin{aligned}
M(a)(0,(i, 0)) & :=1, \\
M(a)\left((i, j),\left(i, j+1 \bmod p_{i}\right)\right) & :=1 \quad \text { for all } j \in\left\{0, \ldots, p_{i}-1\right\}, \\
M\left(b_{i}\right)(0,0) & :=1, \\
M\left(b_{i}\right)((i, j), 0) & :=1 \quad \text { for all } j \in\left\{0, \ldots, p_{i}-1\right\},
\end{aligned}
$$

and setting all other entries of $M(a), M\left(b_{1}\right), \ldots, M\left(b_{m}\right)$ to 0 ; see Figure 3.

We have $M\left(\Sigma^{*}\right) \subseteq\{0,1\}^{Q \times Q}$, i.e., $M\left(\Sigma^{*}\right)$ is an unambiguous monoid of relations. For all $q \in Q$ and all $q^{\prime} \in Q \backslash\{0\}$ we have $M\left(b_{1}\right)\left(q, q^{\prime}\right)=0$, i.e., $M\left(b_{1}\right)$ has rank 1. For 
all $w \in \Sigma^{*}$ there is $q \in Q$ with $M(w)(0, q)=1$, i.e., 1 is the minimum rank in $M\left(\Sigma^{*}\right)$. A shortest word $w_{0} \in \Sigma^{*}$ such that $M\left(w_{0}\right)$ has rank 1 and $M\left(w_{0}\right)\left(0,\left(i, p_{i}-1\right)\right)=1$ holds for all $i \in\{1, \ldots, m\}$ is the word $w_{0}=b_{1} a^{P}$, where $P=\prod_{i=1}^{m} p_{i} \geq 2^{m}$. On the other hand, we have $|Q|=1+\sum_{i=1}^{m} p_{i} \in O\left(m^{2} \log m\right)$ by the prime number theorem.

Hence there is no polynomial $p$ such that $P \leq p(|Q|)$ holds for all $m$.

\section{REFERENCES}

[1] D. Ananichev and V. Vorel, A new lower bound for reset threshold of binary synchronizing automata with sink, J. Autom. Lang. Comb., 24 (2019), pp. 153-164.

[2] P. Bell, M. Hirvensalo, and I. Potapov, Mortality for $2 \times 2$ matrices is NP-hard, in Proceedings of Mathematical Foundations of Computer Science (MFCS), Lecture Notes in Comput. Sci. 7464, Springer, Heidelberg, 2012, pp. 148-159.

[3] J. Berstel and D. Perrin, Trends in the theory of codes, Bull. Eur. Assoc. Theor. Comput. Sci. EATCS, 29 (1986), pp. 84-95.

[4] J. Berstel, D. Perrin, And C. Reutenauer, Codes and Automata, Encyclopedia Math. Appl. 129, Cambridge University Press, Cambridge, 2010.

[5] A. CARPI, On synchronizing unambiguous automata, Theoret. Comput. Sci., 60 (1988), pp. 285-296.

[6] A. Carpi and F. D'Alessandro, On incomplete and synchronizing finite sets, Theoret. Comput. Sci., 664 (2017), pp. 67-77.

[7] I. Daubechies and J. Lagarias, Corrigendum/addendum to: Sets of matrices all infinite products of which converge, Linear Algebra Appl., 327 (2001), pp. 69-83.

[8] P. Goralčík, Z. Hedrlín, V. Koubek, and J. RYšlinková, A game of composing binary relations, RAIRO Inform. Théor., 16 (1982), pp. 365-369.

[9] V. Gusev and E. Pribavkina, On non-complete sets and Restivo's conjecture, in Proceedings of Developments in Language Theory (DLT), Lecture Notes in Comput. Sci. 6795, Springer, Heidelberg, 2011, pp. 239-250.

[10] V. Halava, T. Harju, and M. Hirvensalo, Undecidability bounds for integer matrices using Claus instances, Internat. J. Found. Comput. Sci., 18 (2007), pp. 931-948.

[11] R. Horn and C. Johnson, Matrix Analysis, 2nd ed., Cambridge University Press, Cambridge, 2013.

[12] S. Julia, A. Malapert, and J. Provillard, A synergic approach to the minimal uncompletable words problem, J. Autom. Lang. Comb., 22 (2017), pp. 271-286.

[13] R. Jungers, V. Protasov, And V. Blondel, Efficient algorithms for deciding the type of growth of products of integer matrices, Linear Algebra Appl., 428 (2008), pp. 2296-2311.

[14] J.-Y. KaO, N. Rampersad, And J. Shallit, On NFAs where all states are final, initial, or both, Theoret. Comput. Sci., 410 (2009), pp. 5010-5021.

[15] P. MaRTugin, A series of slowly synchronizing automata with a zero state over a small alphabet, Inform. and Comput., 206 (2008), pp. 1197-1203.

[16] M. Mika and M. SzykuŁa, Complexity of the Frobenius Monoid Problem for a Finite Language, preprint, https://arxiv.org/abs/1902.06702, 2019.

[17] M. Paterson, Unsolvability in $3 \times 3$ matrices, Studies in Appl. Math., 49 (1970), pp. 105-107.

[18] I. Potapov and P. Semukhin, Decidability of the membership problem for $2 \times 2$ integer matrices, in Proceedings of the 2017 Annual ACM-SIAM Symposium on Discrete Algorithms (SODA), SIAM, Philadelphia, 2017, pp. 170-186, https://doi.org/10.1137/1. 9781611974782.12 .

[19] A. Restivo, Some remarks on complete subsets of a free monoid, in Noncommutative Structures in Algebra and Geometric Combinatorics, Quad. "Ricerca Sci." 109, CNR, Rome, 1981, pp. 19-25.

[20] A. Ryzhikov And M. SzykuŁA, Finding short synchronizing words for prefix codes, in Proceedings of the 43rd International Symposium on Mathematical Foundations of Computer Science, LIPIcs. Leibniz Int. Proc. Inform. 117, Schloss Dagstuhl. Leibniz-Zent. Inform., Wadern, 21.

[21] M.-P. Schützenberger, On the definition of a family of automata, Information and Control, 4 (1961), pp. 245-270.

[22] W. TZEnG, A polynomial-time algorithm for the equivalence of probabilistic automata, SIAM

Copyright $@$ by SIAM. Unauthorized reproduction of this article is prohibited. 
J. Comput., 21 (1992), pp. 216-227, https://doi.org/10.1137/0221017.

[23] M. Volkov, Synchronizing automata and the Černý conjecture, in Language and Automata Theory and Applications, Lecture Notes in Comput. Sci. 5196, Springer, Berlin, 2008, pp. $11-27$.

[24] A. Weber And H. Seidl, On finitely generated monoids of matrices with entries in $\mathbb{N}$, RAIRO Inform. Théor. Appl., 25 (1991), pp. 19-38.

Copyright (c) by SIAM. Unauthorized reproduction of this article is prohibited. 